\title{
Relativistic Shock Waves and Mach Cones in Viscous Gluon Matter
}

\author{
loannis Bouras ${ }^{* a}$ Etele Molnár ${ }^{b c}$ Harri Niemi, ${ }^{b}$ Zhe Xu, ${ }^{a b}$ Andrej El, ${ }^{a}$ Oliver Fochler, ${ }^{a}$ \\ Francesco Lauciello, ${ }^{a}$ Felix Reining, ${ }^{a}$ Christian Wesp, ${ }^{a}$ Carsten Greiner ${ }^{a}$ and Dirk \\ H. Rischke ${ }^{a b}$ \\ ${ }^{a}$ Institut für Theoretische Physik, Johann Wolfgang Goethe-Universität \\ Max-von-Laue-Str. 1, D-60438 Frankfurt am Main, Germany \\ ${ }^{b}$ Frankfurt Institute for Advanced Studies \\ Ruth-Moufang-Str. 1, D-60438 Frankfurt am Main, Germany \\ ${ }^{c}$ KFKI, Research Institute of Particle and Nuclear Physics \\ H-1525 Budapest, P.O.Box 49, Hungary \\ E-Mail: bouraseth.physik.uni-frankfurt.de
}

\begin{abstract}
To investigate the formation and the propagation of relativistic shock waves in viscous gluon matter we solve the relativistic Riemann problem using a microscopic parton cascade. We demonstrate the transition from ideal to viscous shock waves by varying the shear viscosity to entropy density ratio $\eta / s$. Furthermore we compare our results with those obtained by solving the relativistic causal dissipative fluid equations of Israel and Stewart (IS), in order to show the validity of the IS hydrodynamics. Employing the parton cascade we also investigate the formation of Mach shocks induced by a high-energy gluon traversing viscous gluon matter. For $\eta / s=0.08$ a Mach cone structure is observed, whereas the signal smears out for $\eta / s \geq 0.32$.
\end{abstract}

XLVIII International Winter Meeting on Nuclear Physics, BORMIO2010

January 25-29, 2010

Bormio, Italy

* Speaker. 


\section{Introduction}

Jet quenching has been discovered in heavy-ion collisions at BNL's Relativistic Heavy Ion Collider (RHIC). In this context, very exciting jet-associated particle correlations [2] have been observed, which indicates the formation of shock waves in the form of Mach cones [3], induced by supersonic partons moving through the quark-gluon plasma (QGP). Measuring the Mach cone angle could give us the possibility to extract the equation of state of the QGP.

Shock waves can only develop in a medium which behaves like a fluid. The large elliptic flow coefficient $v_{2}$ measured at RHIC [4] implies that the QGP created could be a nearly perfect fluid with a small viscosity. Calculations of viscous hydrodynamics [5] and microscopic transport theory [6] have estimated the shear viscosity to the entropy density ratio $\eta / s$ to be less than 0.4 . There is still an open question if this upper limit of the $\eta / s$ ratio is small enough to allow the formation of Mach shocks.

In this work we address the question, whether and when relativistic shock waves and Mach cones can develop in viscous gluon matter for given $\eta / s$ values. For this purpose we consider first the relativistic Riemann problem [7], which we solve within the kinetic theory and the IsraelStewart (IS) theory of viscous hydrodynamics for comparison. Here we employ the microscopic parton cascade BAMPS (Boltzmann Approach of MultiParton Scatterings) [8] and a solver of the IS equations, vSHASTA (viscous SHArp and Smooth Transport Algorithm) [9]. Particularly, we demonstrate agreement between the two approaches for matter with (extreme) small $\eta / s$ values and also show deviations when the $\eta / s$ ratio becomes large, which implies the invalidity of the IS theory. Second, we consider a traverse of a high-energy gluon through gluon matter and investigate the formation of shocks in form of Mach cones. Preliminary results are obtained by using BAMPS.

\section{BAMPS and vSHASTA}

BAMPS is a microscopic transport model solving the Boltzmann equation

$$
p^{\mu} \partial_{\mu} f(x, p)=C(x, p)
$$

for on-shell particles with the collision integral $C(x, p)$. The algorithm for collisions is based on the stochastic interpretation of the transition rate [8]. In this study, we consider only binary gluon scattering processes with an isotropic cross section, which is adjusted locally at each time step to keep a constant $\eta / s$ value $[10,11]$.

vSHASTA solves the IS equations of dissipative hydrodynamics for shear pressure and heat flow. In 1+1 dimensions the relaxation equations for heat conductivity and shear stress are

$$
\begin{aligned}
& D q^{z}=\frac{1}{\tau_{q}}\left(q_{N S}^{z}-q^{z}\right)-I_{q 1}^{z}-I_{q 2}^{z}-I_{q 3}^{z}, \\
& D \pi=\frac{1}{\tau_{\pi}}\left(\pi_{N S}-\pi\right)-I_{\pi 1}-I_{\pi 2}-I_{\pi 3},
\end{aligned}
$$

where $\pi_{N S}$ and $q_{N S}^{z}$ are the Navier-Stokes values [9] and $D \equiv u^{\mu} \partial_{\mu} . \tau_{q}$ and $\tau_{\pi}$ are the relaxation times, respectively. The terms $I_{q i}^{z}$ and $I_{\pi i}$ are explicitly given in [9]. For vanishing shear pressure and heat conductivity the IS equations reduce to the equations of ideal hydrodynamics. 


\section{The relativistic Riemann problem}

The relativistic Riemann problem $[7,10]$ is a well-known shock tube problem in ideal hydrodynamics. For a perfect fluid, i.e., $\eta / s=0$, we observe (the green curve in Fig. 1) in longitudinal direction $z$ a propagation of a shock wave to the right with a larger velocity than the speed of sound and a rarefaction wave to the left exactly with the speed of sound $c_{s}=1 / \sqrt{3}$, where $e=3 P$. In transverse direction the problem is homogeneous.
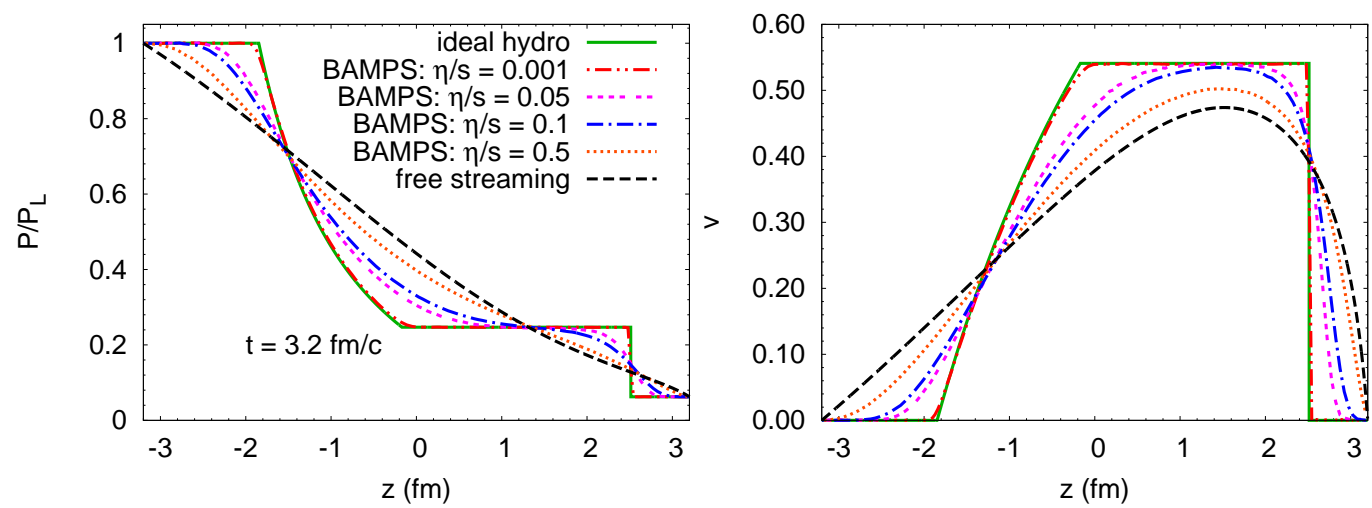

Figure 1: Spatial profile of pressure (left panel, normalized by the initial value $P_{L}$ ) and collective velocity (right panel) at $t=3.2 \mathrm{fm} / \mathrm{c}$. Initially matter is separated in two regions, $z_{L} \leq 0$ and $z_{R}>0$, with two different pressures $P_{L}=5.428 \mathrm{GeV} / \mathrm{fm}^{3}$ and $P_{R}=0.339 \mathrm{GeV} / \mathrm{fm}^{3}$.

The BAMPS solutions for various $\eta / s$ are depicted in Fig. 1. In particular, the BAMPS result for $\eta / s=0.001$ reproduces the ideal solution for a perfect fluid to a very high precision. With the increasing $\eta / s$ value we see a clear transition from the formation of shock waves in ideal fluid to the smearing out in free streaming of particles [10]. The characteristic shock profiles disappear for large $\eta / s$ values.
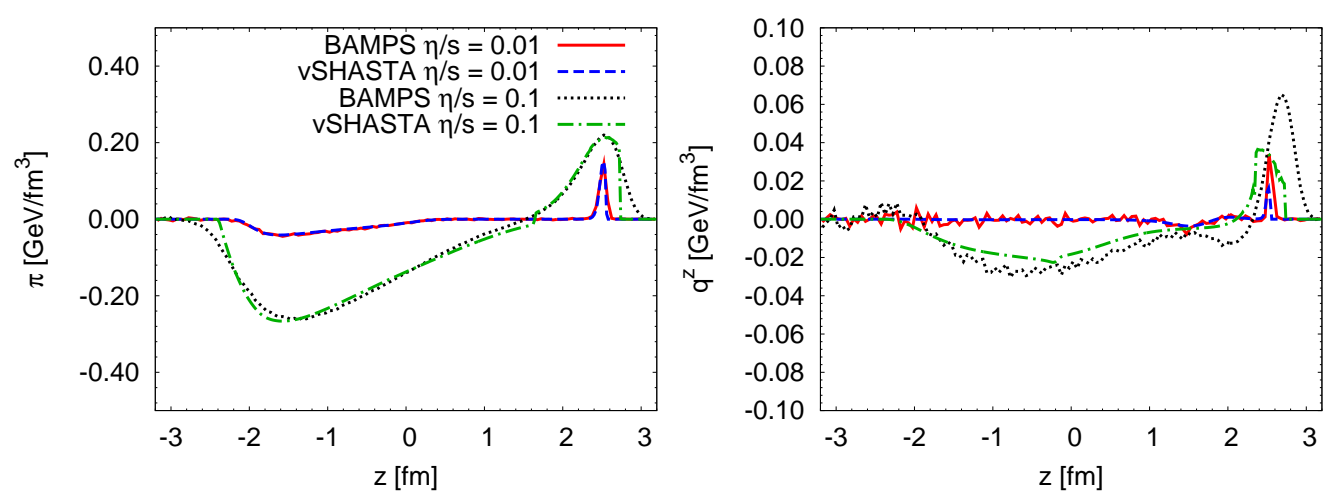

Figure 2: Comparison of BAMPS and vSHASTA for the shear pressure $\pi$ and heat flow $q^{z}$.

In Fig. 2 we show comparisions between BAMPS and vSHASTA for the shear pressure $\pi=$ $\pi^{z z} / \gamma^{2}$ and heat flow $q^{z}=h \gamma^{2}\left(v N^{0}-N^{3}\right)$ at $t=3.2 \mathrm{fm} / \mathrm{c} . \pi^{\mu v}$ is the shear stress tensor, $N^{\mu}$ is the particle four-flow, $\gamma^{2}=\left(1-v^{2}\right)^{-1}$ and $h=(e+P) / n$ is the enthalpy per particle. For $\eta / s=0.01$ we see a very good agreement between vSHASTA and BAMPS, whereas for $\eta / s=0.1$ deviations in 
the region of the shock front and rare faction fan appear. In the case of $\eta / s=0.1$, the local system at the shock front and rare faction fan is strongly out of equilibrium and, thus, the applicability of the IS theory is questionable. The microscopic transport approach does not suffer from this drawback.

\section{Mach Cones in BAMPS}

Formation of 3-dimensional shock waves in form of Mach cones is investigated by shooting a gluon with energy of $20 \mathrm{GeV}$ into a thermal gluon medium with a temperature of $T=400 \mathrm{MeV}$. The thermal medium is embedded in a static box.
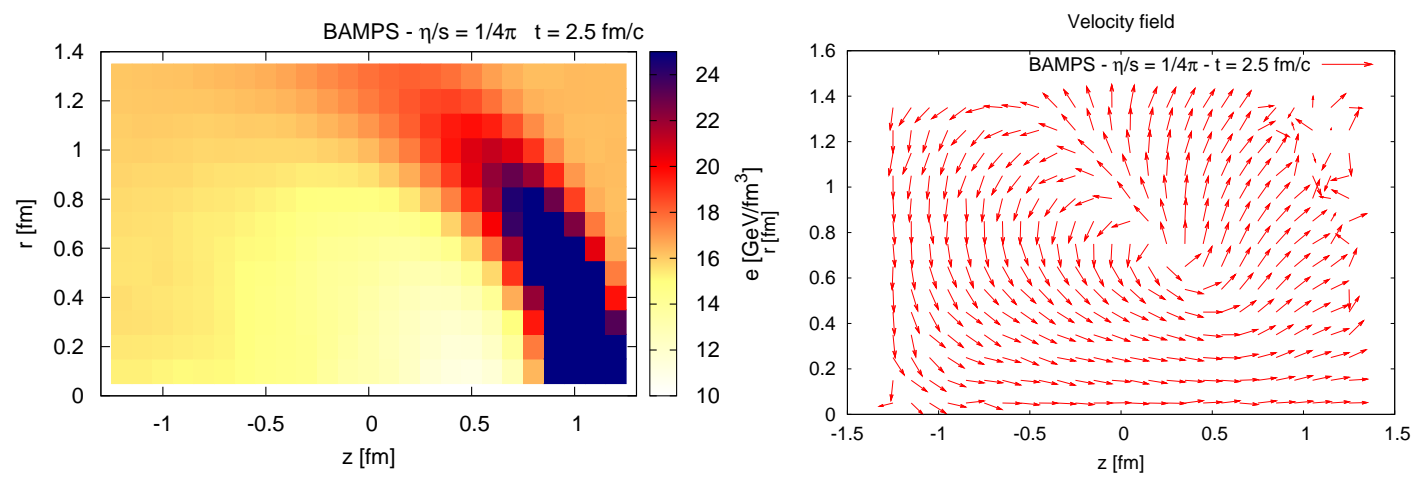

Figure 3: Spatial profile of the energy density (left panel) and the velocity field (right panel). The $\eta / s$ value of the thermal medium is $1 / 4 \pi$. The initial velocity of the high-energy gluon is in z-direction. The length of the arrays in the right panel is unit, i.e., only the direction of the velocity is shown.

Fig. 3 shows spatial profiles of the energy density and velocity field at $t=2.5 \mathrm{fm} / \mathrm{c}$ for a medium with $\eta / s=1 / 4 \pi$. The energy, which the gluon probe lost due to interactions with the medium, creates a shock wave that propagates in form of a Mach cone. The energy density of the region behind the Mach cone is smaller than the initial energy density of the medium. This region is called a diffusion wake. Collective behavior of the medium response is also clearly seen in the profile of the velocity field. Our results agree qualitatively with those found in [12].

For higher values of the $\eta / s$ ratio the typical Mach cone structure smears out as observed in Fig. 4. The strength of the Mach cone signal and also the lower energy density region behind the shock front become weaker because of weaker particle interactions in medium with larger $\eta / s$.

\section{Acknowledgements}

The authors are grateful to the Center for the Scientific Computing (CSC) at Frankfurt for the computing resources. I. Bouras is grateful to HGS Hire. E. Molnár acknowledges the Return Fellowship support by the Alexander von Humboldt foundation. The work of H. Niemi was supported by the Extreme Matter Institute (EMMI).

This work was supported by the Helmholtz International Center for FAIR within the framework of the LOEWE program launched by the State of Hesse. 

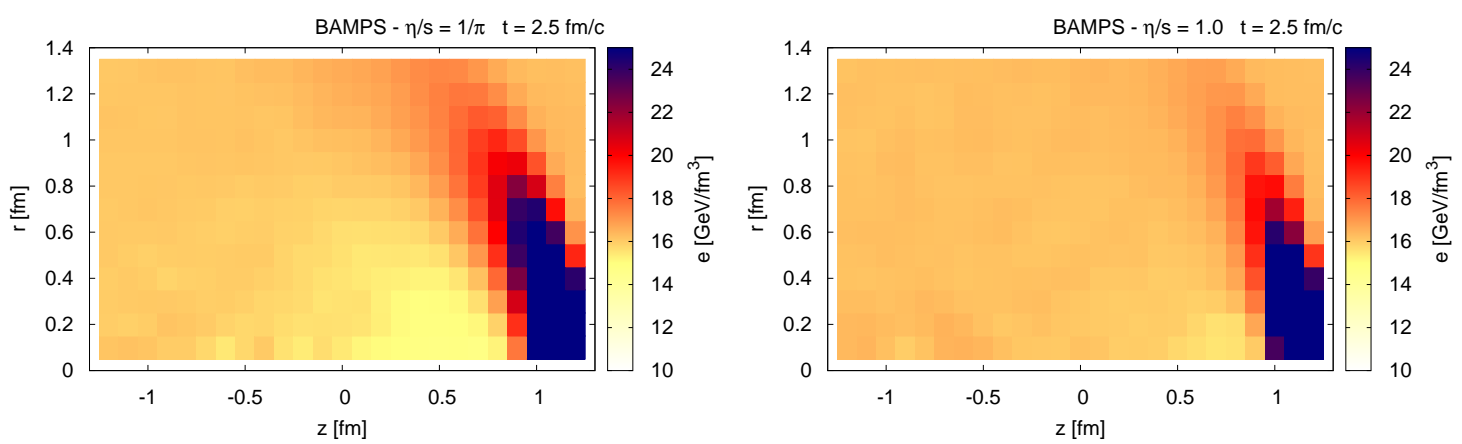

Figure 4: Same as Fig. 3, but for $\eta / s=1 / \pi$ (left panel) and 1.0 (right panel).

\section{References}

[1] J. Adams et al. [STAR Collaboration], Phys. Rev. Lett. 91, 172302 (2003); A. Adare et al. [PHENIX Collaboration], ibid. 101, 232301 (2008).

[2] F. Wang [STAR Collaboration], J. Phys. G 30, S1299 (2004); J. Adams et al. [STAR Collaboration], Phys. Rev. Lett. 95, 152301 (2005); S. S. Adler et al. [PHENIX Collaboration], ibid. 97, 052301 (2006); J. G. Ulery [STAR Collaboration], Nucl. Phys. A 774, 581 (2006); N. N. Ajitanand [PHENIX Collaboration], ibid. 783, 519 (2007); A. Adare et al. [PHENIX Collaboration], Phys. Rev. C 78, 014901 (2008).

[3] H. Stöcker, Nucl. Phys. A 750, 121 (2005); J. Ruppert and B. Müller, Phys. Lett. B 618, 123 (2005); J. Casalderrey-Solana, E. V. Shuryak and D. Teaney, J. Phys. Conf. Ser. 27, 22 (2005); V. Koch, A. Majumder and X. N. Wang, Phys. Rev. Lett. 96, 172302 (2006).

[4] S. S. Adler et al. [PHENIX Collaboration], Phys. Rev. Lett. 91, 182301 (2003); J. Adams et al. [STAR Collaboration], ibid. 92, 052302 (2004); B. B. Back et al. [PHOBOS Collaboration], Phys. Rev. C 72, 051901 (2005).

[5] M. Luzum and P. Romatschke, Phys. Rev. C 78, 034915 (2008); H. Song and U. W. Heinz, arXiv:0812.4274.

[6] Z. Xu, C. Greiner and H. Stöcker, Phys. Rev. Lett. 101, 082302 (2008); Z. Xu and C. Greiner, Phys. Rev. C 79, 014904 (2009).

[7] V. Schneider et al., J. Comput. Phys. 105 (1993) 92.

[8] Z. Xu and C. Greiner, Phys. Rev. C 71 (2005) 064901 [arXiv:hep-ph/0406278].

[9] E. Molnar, H. Niemi and D. H. Rischke, Eur. Phys. J. C 65 (2010) 615 [arXiv:0907.2583 [nucl-th]].

[10] I. Bouras et al., Phys. Rev. Lett. 103 (2009) 032301 I. Bouras et al., arXiv:1006.0387 [hep-ph].

[11] Z. Xu and C. Greiner, Phys. Rev. Lett. 100, 172301 (2008); A. El et al., Phys. Rev. C 79, 044914 (2009)

[12] B. Betz et al., arXiv:0812.4401; D. Molnar, AIP Conf. Proc. 1182, 791 (2009) [arXiv:0908.0299 [nucl-th]]. 